INPLASY

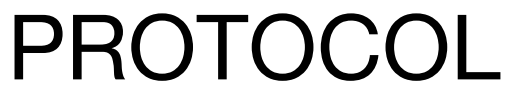

To cite: Chen et al.

Assessment of the efficacy and safety of traditional Chinese medicine therapy for gastric cancer A protocol for systematic review and metaanalysis. Inplasy protocol 2020110120. doi:

10.37766/inplasy2020.11.0120

Received: 27 November 2020

Published: 27 November 2020

Corresponding author:

Zhiqun Cao

cheerup_u@163.com

Author Affiliation:

The First Clinical College, Shandong University of Traditional Chinese Medicine

Support: NSFY(81904128).

Review Stage at time of this submission: The review has not yet started.

Conflicts of interest:

None.

\section{Assessment of the efficacy and safety of traditional Chinese medicine therapy for gastric cancer A protocol for systematic review and meta-analysis}

Chen, T1; Cao, Z².

Review question / Objective: This review aims to systematically evaluate the efficacy and safety of traditional chinese medicine for gastric cancer reported in randomized clinical trials (RCTs).

Condition being studied: Gastric cancer: digestive system malignant tumors.

Information sources: PubMed, EMBASE, the Cochrane Central Register of Controlled Trials (CENTRAL), CNKI (Chinese National Knowledge Infrastructure), and CBM (Chinese Biological Medical Database).

INPLASY registration number: This protocol was registered with the International Platform of Registered Systematic Review and Meta-Analysis Protocols (INPLASY) on 27 November 2020 and was last updated on 27 November 2020 (registration number INPLASY2020110120).

\title{
INTRODUCTION
}

Review question / Objective: This review aims to systematically evaluate the efficacy and safety of traditional chinese medicine for gastric cancer reported in randomized clinical trials (RCTs).
Condition being studied: Gastric cancer :digestive system malignant tumors.

\section{METHODS}

Participant or population: Patients with GC (diagnosed and classified as proposed by 
NCCN guideline ) without age, gender, and racial limitations.

Intervention: TCM were used exclusively or in combination with other therapeutic methods. Types of Chinese medicines, combined use methods will be ignored.

Comparator: Other therapeutic methods (include the any other non-TCM treatment) or in combination with sham TCM.

Study designs to be included: This study includes all relevant randomized controlled trials (RCTs) of traditional chinese medicine therapy for GC published in Chinese or English, regardless of allocation hidden or blinded.

Eligibility criteria: Randomized controlled trials (RCTs) will be included in this study. There were no limitations on year of publication, publication status.

Information sources: PubMed, EMBASE, the Cochrane Central Register of Controlled Trials (CENTRAL), CNKI (Chinese National Knowledge Infrastructure), and CBM (Chinese Biological Medical Database).

Main outcome(s): Karnofsky Performance Scale score, quality of Life.

Quality assessment / Risk of bias analysis: The risk of bias of included nonrandomized studies will be evaluated according to the tool for assessing risk of bias in nonrandomized studies of interventions (ROBINS-I).

Strategy of data synthesis: Pairwise metaanalyses. Using Revman 5.3 software provided by Cochrane Collaboration Network, meta-analyses were carried out for the included researches.

Subgroup analysis: Subgroup analyses are designed for age, sex, and different preoperative chemotherapy methods.

Sensibility analysis: Assess the sensitivity of results by analyzing studies including patients without complications, the studies without missing data.

Country(ies) involved: China.

Keywords: Gastric cancer; meta-analysis, protocol, systematic review, traditional Chinese medicine.

Contributions of each author:

Author 1 - Ting Chen.

Email: cheerup_u@163.com

Author 2 - Zhiqun Cao.

Email: cheerup_u@163.com 